\title{
Simultaneous neutron transmission and diffraction contrast tomography as a non-destructive 3D method for bulk single crystal quality investigations
}

\author{
S. Peetermans ${ }^{1,2, a)}$ and E. H. Lehmann ${ }^{1}$ \\ ${ }^{1}$ Paul Scherrer Institut, Neutron Imaging and Activation Group, CH-5232 Villigen PSI, Switzerland \\ ${ }^{2}$ Ecole polytechnique fédérale de Lausanne, NXMM laboratory, IMX, CH-1015, Lausanne, Switzerland
}

(Received 15 August 2013; accepted 12 September 2013; published online 30 September 2013)

\begin{abstract}
Traditional neutron tomography allows to reconstruct the attenuation cross section, a measure for the material distribution, at high spatial resolution and non-destructively. However, it does not state anything about the ordering structure of the atoms inside this material. Extending the setup with a second neutron imaging detector, diffracted neutrons from the ordered crystal lattice could be captured. Emerging iterative reconstruction techniques allow reconstructing the local Bragg reflectivity in the sample, a measure for the spatial distribution in crystal quality (orientation, homogeneity of phases). Simultaneous acquisition ensures optimal use of the neutron flux and a direct comparison of different sample properties. (C) 2013 AIP Publishing LLC. [http://dx.doi.org/10.1063/1.4823741]
\end{abstract}

\section{INTRODUCTION}

Neutron imaging ${ }^{1,2}$ traditionally relies on the attenuation of a polychromatic neutron beam passing through a sample of interest. It is thus sensitive to differences in sample material and thickness, which can be separated spatially making a tomography from radiographies acquired for multiple sample rotation angles. Though it has solved problems in numerous fields ranging from materials science to paleontology, it is insensitive to the crystal structure for polycrystalline materials as its energy-dependent cross section footprint is averaged out over the full beam spectrum. Energy-selective neutron imaging ${ }^{3}$ mitigates this problem by reducing the spectral bandwidth of the incident beam, though at the cost of increased exposure times by at least one order of magnitude. Moreover, the number of rotation angles for which a single crystal can diffract neutrons out of the monochromatic direct beam, i.e., provide transmission contrast, is limited. In this work, we present 3D reconstruction of a single crystal in a polychromatic neutron beam using the neutrons that are diffracted.

Diffraction imaging or topography has a long history in the X-ray community since Berg obtained his first diffraction projections on rock salt in $1931 .^{4}$ Stimulated by the availability of high-intensity synchrotron radiation beams, a multitude of variations nowadays exists at different dedicated beamlines. ${ }^{5,6}$ One can work with a polychromatic or monochromatic beam that can either fully encompass the sample or be limited to a line section through it. Extension to $3 \mathrm{D}$ also exists with X-ray diffraction tomography methods such as topo-tomography, ${ }^{7} \mathrm{DCT}^{8}{ }^{8}$ and $3 \mathrm{DXRD} .{ }^{9}$ But whereas $\mathrm{X}$-rays interact with the electron shell of the atom, neutrons interact with the nucleus, yielding techniques similar in principle, though complementary in practice: neutrons generally offer bulk penetrability of metals, e.g., lead crystals. Neutron topography has been carried out mainly in the ' $70 \mathrm{~s}$ and ' $80 \mathrm{~s}$ using X-ray films with a gadolinium convertor foil as also used for neutron radiography at that time. ${ }^{10}$

\footnotetext{
a) Author to whom correspondence should be addressed. Electronic mail: steven.peetermans@psi.ch
}

The advent of digital methods at the dawn of the new millennium revolutionized neutron imaging and brought routine tomographic investigations. In this work, it is now extended to diffraction imaging and tomography using advanced algebraic reconstruction algorithms. A new set-up was developed for simultaneous high resolution transmission and diffraction tomography, applied to an iron single crystal to find good reconstruction agreement, paving the way for future extension to polycrystalline samples.

\section{EXPERIMENTAL SET-UP}

\section{A. Neutron imaging}

The experiment was conducted at the ICON beamline for imaging with cold neutrons at the Paul Scherrer Institut. ${ }^{11}$ Neutrons are generated through spallation reactions by impact of a $590 \mathrm{MeV}, 1.5 \mathrm{~mA}$ proton beam on a lead target and subsequently moderated in a tank of $\mathrm{D}_{2} \mathrm{O}$ at room temperature and a smaller vessel of liquid $\mathrm{D}_{2}$ at $25 \mathrm{~K}$. The beamline is also equipped with various wavelength selection systems to limit the incident wavelength band, though the full polychromatic spectrum or white beam was used during the experiment. After passing through a sample, neutrons are detected using a scintillator screen that converts them to visible light, which is then guided via a mirror to a digital camera on top, out of the direct beam to protect it from radiation damage. Various combinations of scintillators (material and thickness), light focusing optics and camera's allow for imaging at different resolution and field of view. Two systems are present according to that principle: a "midi"-setup for medium resolution and a "micro" set-up offering the currently highest possible spatial resolution. ${ }^{12}$

\section{B. The double detector set-up}

Transmission and diffraction imaging pose somewhat different detector demands. Transmission imaging is performed in the direct forward beam direction at the highest resolution possible. The diffracted neutron signal on the other hand does not follow the incident neutron beam direction 
TABLE I. Smallest two diffraction angles (with respect to the transmitted beam direction) formed by the two crystal planes of largest interplanar distance for typical engineering materials at the average wavelength of $3.1 \AA$ of the ICON beamline.

\begin{tabular}{lcc}
\hline \hline & 1st reflection & 2nd reflection \\
\hline $\mathrm{Al}$ & $83.1^{\circ}$ & $99.9^{\circ}$ \\
$\mathrm{Cu}$ & $95.9^{\circ}$ & $118.1^{\circ}$ \\
$\alpha-\mathrm{Fe}$ & $99.8^{\circ}$ & - \\
$\mathrm{Ni}$ & $99.3^{\circ}$ & $123.2^{\circ}$ \\
\hline \hline
\end{tabular}

anymore. In a cold neutron beam, the diffraction directions tend towards side and backscattering, illustrated in Table I.

Moreover, a large field of view is required to cover sufficient angular range at the cost of a loss in resolution. A solution to these conflicting demands has been found using a double detector set-up, combining the micro set-up for high resolution transmission imaging in the beam direction and the midi set-up on a movable stage aside from the direct beam, facing the sample, for diffraction imaging. Further developments are made to extend the diffraction imaging range to also include back scattering. Camera trigger and feedback signals are logically combined and the whole is emulated as a single virtual camera in the beamline operating system. The set-up thus allows for simultaneous recording of transmitted and diffracted neutrons, making optimal use of the available beam time and providing easy sample aligning. The different imaging parameters used during this experiment are listed in Table II.

The sample was a cube shaped iron single crystal, measuring $4 \mathrm{~mm} \times 4 \mathrm{~mm} \times 4 \mathrm{~mm}$. A tomography was made rotating it over $360^{\circ}$ in 376 steps using a white beam. The distance between sample and diffraction imaging system was $70 \mathrm{~mm}$. The set-up is depicted schematically in Figure 1.

\section{METHOD}

\section{A. Recorded signal components}

The in-beam detector records the attenuation of the neutron beam as it passes through the sample, following to a good approximation the Lambert-Beer law:

$$
I=I_{0} e^{-\int_{d} \Sigma d x},
$$

$I$ being the recorded intensity profile, $I_{0}$ the incident beam profile on the sample and $\Sigma$ the material and density dependent macroscopic cross-section averaged over the incident

TABLE II. Parameters of the neutron imaging systems used simultaneously in the transmission and diffraction tomography.

\begin{tabular}{lcc}
\hline \hline & Transmission & Diffraction \\
\hline Camera angle & $0^{\circ}$ & $90^{\circ}$ \\
\# Pixels & $2048 \times 2048$ & $2160 \times 2560$ \\
Pixelsize & $13.5 \mu \mathrm{m}$ & $68.3 \mu \mathrm{m}$ \\
Field of view & $27 \times 27 \mathrm{~mm}$ & $100 \times 100 \mathrm{~mm}$ \\
Exposure time & $70 \mathrm{~s}$ & $80 \mathrm{~s}$ \\
\hline \hline
\end{tabular}

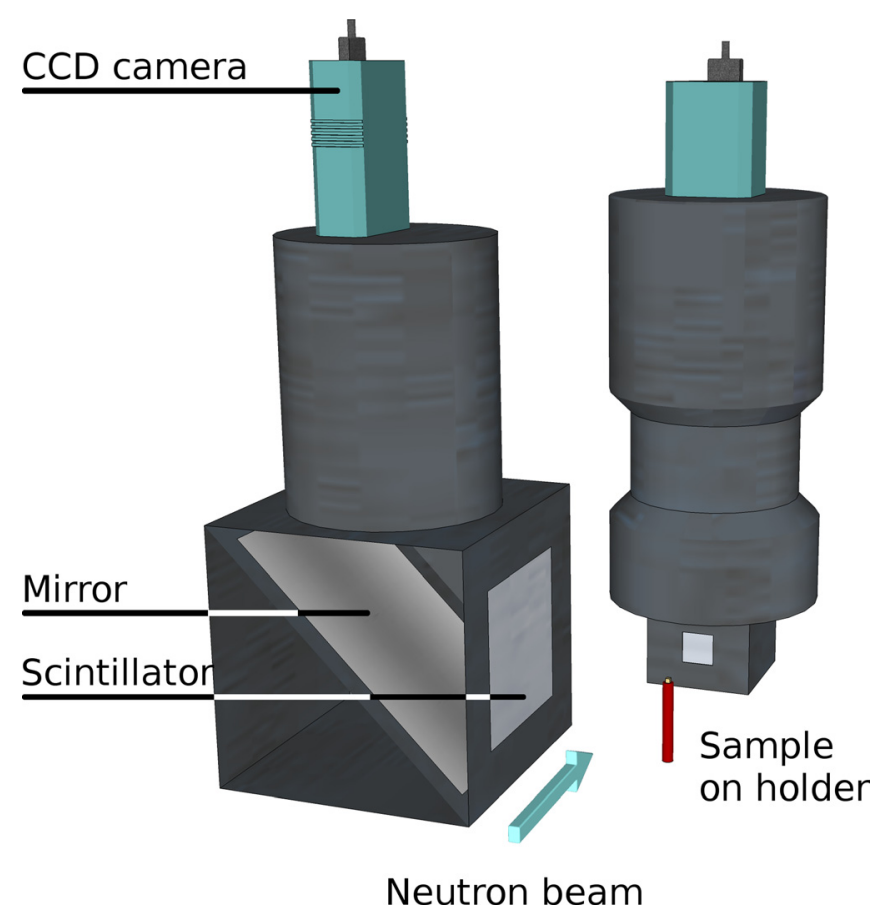

FIG. 1. Schematic overview (to scale) of the double detector set-up with a medium resolution, large field of view neutron imaging system on the left at $90^{\circ}$ with respect to the sample for diffraction imaging and the high resolution, small field of view system in the beam direction behind the sample for transmission imaging.

beam spectrum, the integral of which over the traversed neutron path $d$ through the sample is recorded. Transmission is then defined as $I / I_{0}$, the ratio of the images with and without sample (also called flat fields or open beams).

The intensity recorded with the side detector can be split into two classes. First, coherent elastic scattering from the single crystal sample that will give rise to recorded sample projections via diffraction. Under the approximation of kinematic diffraction, the local diffracted intensity for each point in the crystal adds up along the diffracted beam path through the sample. Changes in crystal quality (defects) induce an increased local scattering power or Bragg reflectivity as also neutrons that do not satisfy the Bragg condition in the crystal matrix can still diffract in the deformed region around the defect. ${ }^{13}$ In the absence of strong internal orientation and strain gradients, parallel diffraction projections are thus formed. They move through the field of view as the sample is rotated, according to Braggs law

$$
2 \mathrm{~d}_{\mathrm{hkl}} \sin (\theta)=\lambda_{\mathrm{hkl}},
$$

where $d_{h k l}$ corresponds to the (hkl) lattice plane spacing, $\theta$ the changing angle between this plane and the incident neutron beam - equal to the angle between the plane and the exiting recorded diffracted beam - and $\lambda_{h k l}$ the wavelength of the neutrons diffracted out of the polychromatic incident beam.

The second component stems from incoherent sample scattering, which is approximately invariant under sample rotation if the sample's horizontal dimension change is small compared to the detector distance, together with scattering from the sample surroundings (air, concrete shielding, 
detection system and sample mount). As it is constant as opposed to the moving first component, it is easily determined for each pixel taking the median over all acquired projections and can subsequently be subtracted to end up with only the sample neutron diffraction projections in our radiographs.

\section{B. Projection segmentation}

Next, all diffraction projections of the sample need to be segmented out of the recorded radiographs as future input for the reconstruction. As the recorded neutron signal is typically weak, this is performed in several steps. Firstly, all pixel regions with a signal to noise ratio (SNR) above a threshold $t_{\text {high }}$ are selected. A size criterion is subsequently applied to distinguish between sample diffraction projections and bright spots formed by gamma rays hitting the camera chip: connected pixel regions should have an area bigger than $S$ pixel $^{2}$. Region growing down to a $t_{\text {low }}$ SNR threshold is then applied in order to also include the less intense parts of the diffraction projection. A closing and smoothing operation finalizes the segmentation. Typical values for these parameters are listed in the results section.

\section{Set-up geometry}

In order to identify at what direction through the sample the diffraction projections segmented previously were taken, their centers of mass at all sample rotations are identified and together with detector position, orientation relative to the sample holder and the sample rotation, these are transformed into a fixed, non-rotating sample coordinate system. The projection direction is then easily found as the vector connecting these points and the crystal's center of mass (determined geometrically for simple samples or from the transmission tomography for more complex sample shapes).

The sample's center of mass position with respect to the side camera needed for the reconstruction was refined further based on Friedl pair registration: if the (hkl) lattice plain fulfills the diffraction condition, then also (-h-k-1) will diffract (i.e., its Friedl pair) after turning the sample $180^{\circ}$. In the sample frame of reference, the two detected Friedl pair diffraction projections form a line through the diffracting crystal. Acquiring multiple Friedl pairs then yields the crystal's center as the intersection of these paths. As the sample rotation angles were not symmetric over $180^{\circ}$ but over $360^{\circ}$, a standard often used in transmission tomography for higher spatial resolution, 20 more diffraction projections were recorded for sample rotation angles in the $180^{\circ}-360^{\circ}$ interval symmetric to angles recorded in the prior tomography scan.

\section{Reconstruction}

The 3D spatial distribution of the macroscopic crosssection is reconstructed by applying the commonly used filtered back projection algorithm on the negative logarithm of the acquired transmission projections. Based on taking the Fourier transform of a projection intensity profile for each rotation angle (or Radon transform) followed by an inverse 2D Fourier transform yielding the spatially resolved reconstruction (Fourier slice theorem), the method is highly efficiently implemented using the fast Fourier transform. ${ }^{14}$

The $3 \mathrm{D}$ distribution of the local scattering power can also be retrieved from its acquired integral footprint in the recorded segmented diffraction projections. Existing transmission imaging reconstruction algorithms can be used if one protracts the diffracted neutron path to a virtual parallel neutron source (see Figure 2 for a schematic impression). A normalization of each projection to its integrated intensity is performed prior to reconstruction as they are all originating from the same diffracting volume, though different projections have different intensities because of different structure factors, spectral distribution of the incident neutron wavelength spectrum and different effective scintillator thicknesses for different diffracted neutron detector incidence angles. To reconstruct the-relative-local scattering power, we apply the three dimensional simultaneous iterative reconstruction technique (3D-SIRT) available in the ASTRA package. ${ }^{15}$ The method relies on rewriting the reconstruction problem as an (underdetermined) matrix equation linking the unknown sample volume to the recorded projections and solving it iteratively. Though computationally expensive, in particular with out of plane acquisitions requiring a full $3 \mathrm{D}$ approach, it offers superior performance dealing with few, non-uniformly spaced acquisition angles. ${ }^{16}$

\section{RESULTS AND DISCUSSION}

Figure 3 shows a typical recorded image on the side detector, background corrected, with two sample diffraction projections segmented (indicated by the red circumference $t_{\text {high }}=5, t_{\text {low }}=2, S=20 \times 20 p l x^{2}$ ).

Figure 4 shows all diffraction projection directions acquired on the unit sphere, with the sample axis system indicated by the (cyan, green, red)-tripod. It clearly shows a

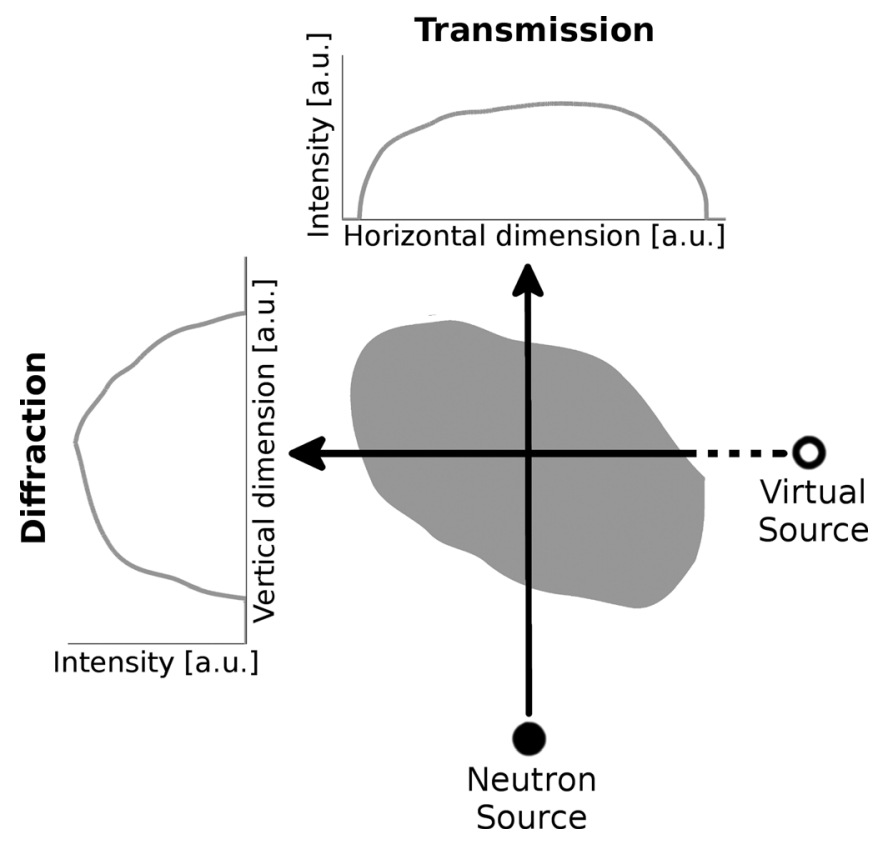

FIG. 2. Schematic overview of the experiment geometry showing acquired neutron transmission signal and the diffraction projection profile that can be assumed to originate from a virtual source. 


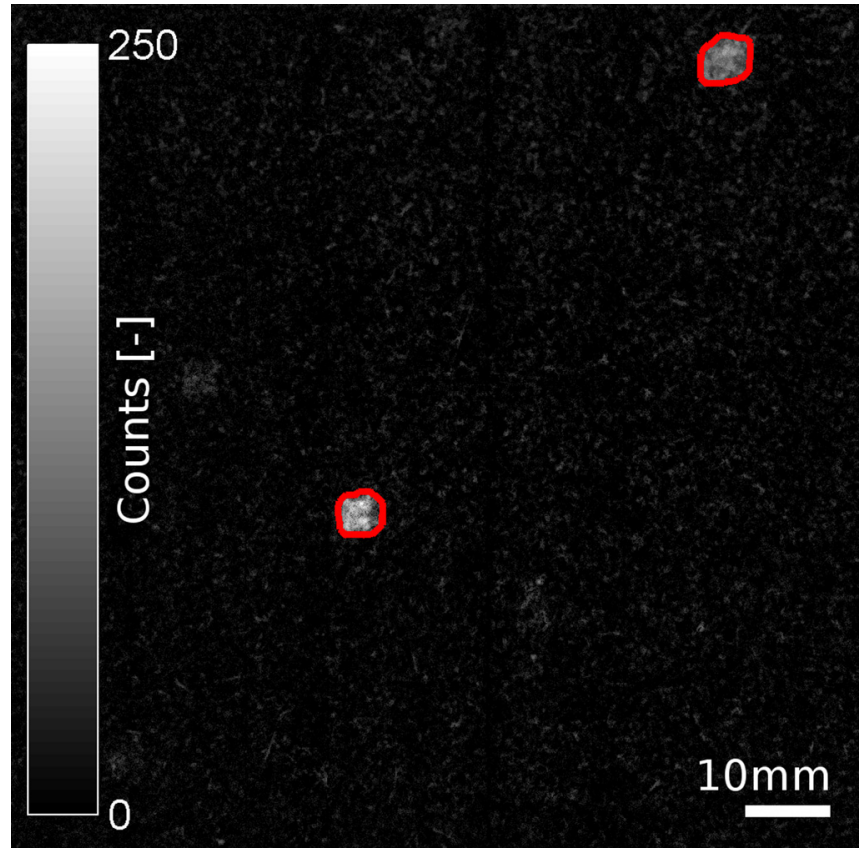

FIG. 3. Background-corrected recorded signal on the side detector, for a sample rotation angle of $95.7^{\circ}$, with segmented sample diffraction projections (outlined in red).

good angular coverage around the sample, however irregular and mostly out of plane requiring a full 3D algebraic reconstruction. The horizontal angular separation is $0.66^{\circ}$ on average, with a maximum of $6.6^{\circ}$.

For the cubic sample in the same position as for Figure 3 , a close up of the two segmented diffraction projections is shown in Figure 5, together with the transmission projection recorded simultaneously. As the sample induces an attenuation of the neutron beam, the transmission projection shows the sample as dark on a white background, whereas the diffraction projections are formed by an increase of detected neutrons and thus bright on a dark background. One can clearly recognize the cube's shape, even though statistics are low.

The subsequent reconstruction based on transmission and diffraction projections is illustrated in Figure 6 by a $3 \mathrm{D}$ volume rendering. The object's shape, orientation and size are reconstructed correctly and consistently. The sample appears homogeneous in its macroscopic cross-section, i.e.,

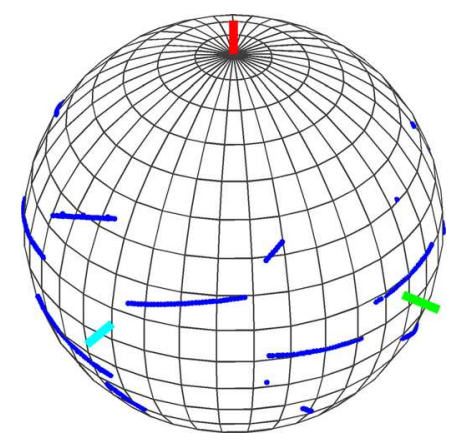

FIG. 4. Segmented diffraction projection directions on the unit sphere in the sample frame of reference, indicated by the (cyan, green, red) tripod. Grid line separation is $10^{\circ}$. a)

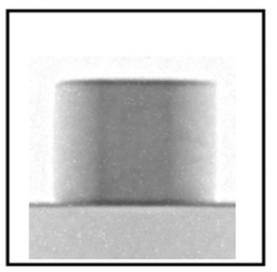

b)

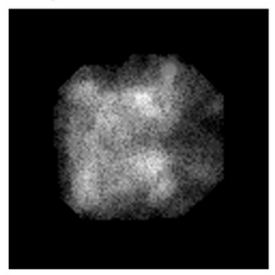

c)

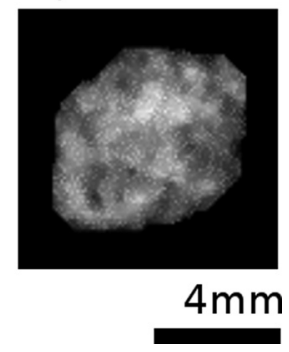

FIG. 5. Transmission projection (a) and diffraction projections of the central (b) and top (c) segmented projections in Figure 3 for the sample at $95.7^{\circ}$.

its material distribution. The reconstructed distribution of local Bragg reflectivity however is higher on one side, implying reduced crystal quality there. Note the polycrystalline sample holder present in the transmission reconstruction and absent in the single crystal diffraction tomography.

A comparison of virtual slices through both reconstructions is given in Figure 7. A distinct blur of the diffraction reconstruction can be observed. This is due to the incident beam divergence and finite source size-characterized in neutron imaging by the beamline's $L / D$, the ratio of source to sample distance over source size-in combination with a certain sample to side detector distance $l$. Though neutron imaging beamlines can to a good approximation be considered parallel for transmission imaging purposes, the standard $L / D=343$ also used in this experiment, together with $l=70 \mathrm{~mm}-99 \mathrm{~mm}$ (diffraction projection in the center of the side detector versus in a corner) yields an observed sample point spread $l /(L / D)=200 \mu \mathrm{m}-290 \mu \mathrm{m}$. Future care should be taken to work at the highest practical beam collimation and lowest sample to side detector distance possible.

A higher local Bragg reflectivity is registered towards the outer edges of the sample. This is however an artifact ascribed to the virtual source assumption applied on a large sample of non-negligible neutron attenuation. As the sourcefacing side of the sample sees higher incident beam intensity

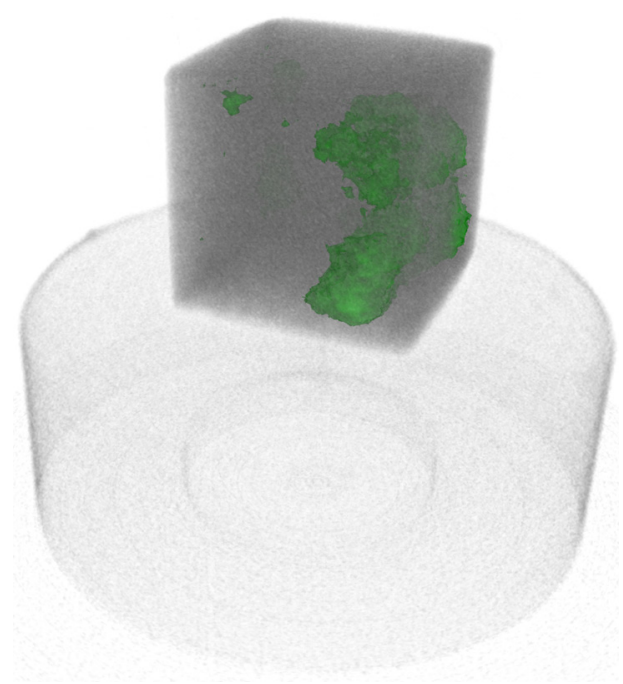

FIG. 6. Volume rendering of the macroscopic cross section as reconstructed from transmission data (gray) and regions of increased local Bragg reflectivity (green). 

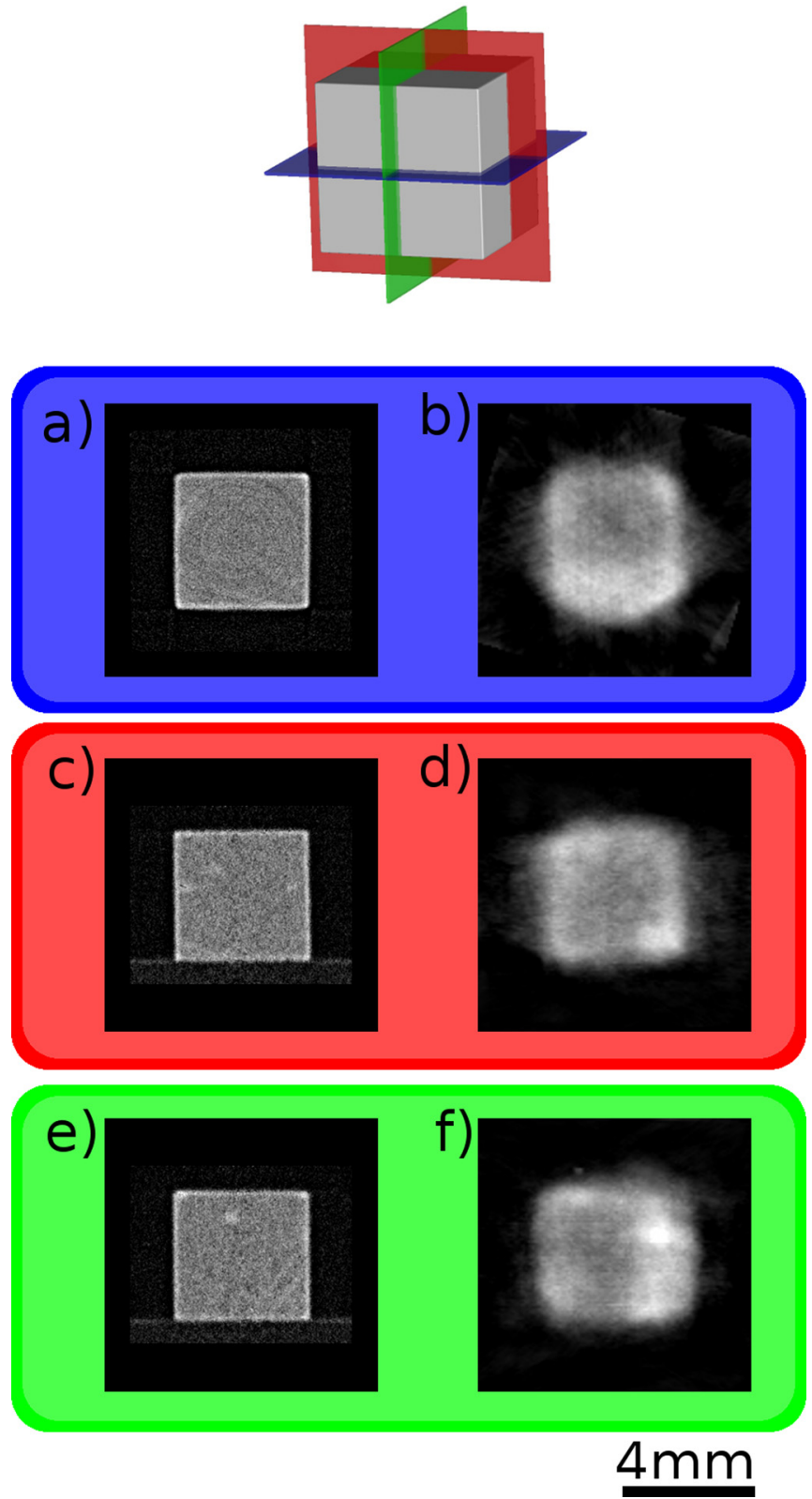

FIG. 7. Horizontal, coronal and sagittal virtual slices through the sample from transmission (a),(c),(e) and diffraction contrast tomography (b),(d),(f).

than the back, also the diffracted intensity coming from this side will be higher. Incorporating all acquired sample orientations will result in lower reconstructed central scattering power compared to the outer edges, much similar to cupping artifacts due to beam hardening in transmission imaging. However, though beyond the scope of this work, the attenuation information contained within the transmission tomography could in principle be included in the diffraction reconstruction.

The used approach bears resemblance to existing monochromatic synchrotron diffraction contrast tomography methods such as topo-tomography ${ }^{7}$ where the local Bragg reflectivity is reconstructed using conventional filtered back projection after tedious alignment of the rotation axis with the diffraction vector so all diffraction projections fall on the same detector position. Methods as $\mathrm{DCT}^{8}$ and $3 \mathrm{DXRD}^{9}$ feature polycrystalline samples rotated around their vertical axis in a monochromatic beam, collecting and grouping the limited number of diffraction projections originating from the different crystallites and reconstructing them iteratively to yield a grain orientation map of the sample. Here, we rotated a single crystal around its vertical axis in a white beam, resulting in many diffraction projections to reconstruct the local Bragg reflectivity from without prior sample alignment and making more efficient use of the far lower neutron flux. An extension to multiple crystallites can be envisaged for neutrons too, either using a monochromator or a ray-tracing approach for discerning between crystallites. Though neutron diffraction contrast tomography resolution is lower than its synchrotron counterpart, a niche can nevertheless be identified for bulk metallic single crystals and large grained samples as in archaeometallurgy, nickel-iron meteorites, neutron monochromator crystals or single crystal superalloy turbine blades.

\section{v. CONCLUSION}

Diffraction contrast based tomography was performed with neutrons for the first time with a double detector setup, delivering information on the spatial distribution in crystal quality. A new set-up was devised to this end, where the traditional transmission neutron tomography set-up is completed with a second imaging detector on the side, recording projections formed by neutrons diffracted from the single crystal sample. After segmentation and determination of the projection direction, the local Bragg reflectivity is reconstructed using advanced iterative reconstruction algorithms. This information can directly be compared and added to the knowledge of the material distribution from the transmission tomography. Applied to a single crystal sample, both transmission and diffraction contrast reconstructions match nicely, though future improvements can still be made in terms of spatial resolution and artifact reduction. Simultaneous acquisition ensures efficient neutron usage, direct comparison of both sample features and future compatibility with dynamic processes, e.g., cracking and crystal growth.

\section{ACKNOWLEDGMENTS}

The author would like to thank W. Ludwig for the fruitful discussions that inspired many of the used evaluation methods and R. Schäfer for providing the sample.

${ }^{1}$ W. Treimer, A. Hilger, N. Kardjilov, and M. Strobl, "Review about old and new imaging signals for neutron computerized tomography," Nucl. Instrum. Methods Phys. Res. A 542, 367-375 (2005).

${ }^{2}$ E. H. Lehmann, A. Kaestner, L. Josic, S. Hartmann, and D. Mannes, "Imaging with cold neutrons," Nucl. Instrum. Methods Phys. Res. A 651, 161-165 (2011).

${ }^{3}$ E. H. Lehmann, G. Frei, L. Josic, N. Kardjilov, A. Hilger, W. Kockelmann, and A. Steuwer, "The energy-selective option in neutron imaging," Nucl. Instrum. Methods Phys. Res. A 603, 429-438 (2009).

${ }^{4}$ W. Berg, "Über eine röntgenographische methode zur untersuchung von gitterstörungen an kristallen," Naturwiss. 19, 391-397 (1931).

${ }^{5}$ B. K. Tanner and D. K. Bowen, "Synchrotron x-radiation topography," Mater. Sci. Eng. R. 8, 369-407 (1992).

${ }^{6}$ J. Baruchel, J. Härtwig, and P. Pernot-Rejmánková, "Present state and perspectives of synchrotron radiation diffraction imaging," J. Synchrotron Radiat. 9, 107-114 (2002). 
${ }^{7}$ W. Ludwig, P. Cloetens, J. Härtwig, J. Baruchel, B. Hamelin, and P. Bastie, "Three-dimensional imaging of crystal defects by 'topotomography'," J. Appl. Crystallogr. 34, 602-607 (2001).

${ }^{8}$ W. Ludwig, P. Reischig, A. King, M. Herbig, and E. M. Lauridsen, "Three-dimensional grain mapping by x-ray diffraction contrast tomography and the use of friedel pairs in diffraction data analysis," Rev. Sci. Instrum. 80, 033905 (2009).

${ }^{9}$ H. F. Poulsen, Three-Dimensional X-Ray Diffraction Microscopy (Springer, 2004).

${ }^{10}$ M. Schlenker and J. Baruchel, "Neutron topography: A review," Physica B 137, 309-319 (1986).

${ }^{11}$ A. P. Kaestner, S. Hartmann, G. Kühne, G. Frei, C. Grünzweig, L. Josic, F. Schmid, and E. H. Lehmann, "The icon beamline-a facility for cold neutron imaging at sinq," Nucl. Instrum. Methods Phys. Res. A 659, 387-393 (2011).
${ }^{12}$ E. H. Lehmann, G. Frei, G. Kuehne, and P. Boillat, "The micro-setup for neutron imaging: A major step forward to improve the spatial resolution," Nucl. Instrum. Methods Phys. Res. A 576(2-3), 389-396 (2007).

${ }^{13}$ D. K. Bowen and B. K. Tanner, High Resolution X-ray Diffractometry and Topography (Taylor \& Francis, 1998).

${ }^{14}$ A. C. Kak and M. Slaney, "Principles of Computerized Tomographic Imaging," in Algorithms for Reconstruction with Nondiffracting Sources (IEEE Press, 1999), pp. 49-112, Chap. 3.

${ }^{15}$ W. J. Palenstijn, K. J. Batenburg, and J. Sijbers, "Performance improvements for iterative electron tomography reconstruction using graphics processing units (gpus)," J. Struct. Biol. 176, 250-253 (2011).

${ }^{16}$ A. C. Kak and M. Slaney, "Principles of computerized tomographic imaging," in Algebraic Reconstruction Algorithms (IEEE Press, 1999), pp. 276-296, Chap. 7. 